\title{
Optimum Bias Point in Broadband Subcarrier Multiplexing with Optical IQ Modulators
}

\author{
Fernando A. Gutiérrez, Philip Perry, Frank Smyth, Andrew D. Ellis and Liam P. Barry
}

\begin{abstract}
This paper develops a theoretical analysis of the tradeoff between carrier suppression and nonlinearities induced by optical IQ modulators in direct-detection subcarrier multiplexing systems. The tradeoff is obtained by examining the influence of the bias conditions of the modulator on the transmitted single side band signal. The frequency components in the electric field and the associated photocurrent at the output of the IQ modulator are derived mathematically. For any frequency plan, the optimum bias point can be identified by calculating the sensitivity gain for every subchannel. A setup composed of subcarriers located at multiples of the data rate ensures that the effects of intermodulation distortion are studied in the most suitable conditions. Experimental tests with up to five QPSK electrical subchannels are performed to verify the mathematical model and validate the predicted gains in sensitivity.
\end{abstract}

Index Terms - Carrier to signal power ratio (CSPR), nonlinear distortion (NLD), optical IQ modulator, subcarrier multiplexing (SCM).

\section{INTRODUCTION}

$\mathrm{O}$ PTICAL subcarrier multiplexing (SCM) relies on the excellent stability and low phase noise of microwave oscillators to generate one or more digitally modulated radiofrequency $(\mathrm{RF})$ signals that are then intensity modulated onto an optical wavelength before transmission over fibre. SCM can be combined with wavelength division multiplexing (WDM) to increase the flexibility of the network. Analogue and digital SCM approaches have been used in many applications, including cable television (CATV) [1], radio over fibre [2] also focusing on the increasingly important Common Public Radio Interface (CPRI) [3], broadband point to point links [4], access networks [5] and $100 \mathrm{Gbit} / \mathrm{s}$ local area networks [6]. In such a system it is desired to guarantee the following features: optical single side band (SSB) to increase spectral efficiency and eliminate dispersive fading [7], low inter channel distortion and an appropriate level of the

Manuscript submitted on October 3, 2014; revised December 2, 2014. This work was supported in part by the EI CFTD grant CF/2011/1627, SFI grants 09/IN.1/12653 and 10/CE/I1853 and EPSRC grant EP/L000091/1.

F. A. Gutierrez, P. Perry, F. Smyth and L. Barry are with The RINCE Institute, Dublin City University, Glasnevin, Dublin 9, Ireland. (E-mail: fernando.gutierrez@dcu.ie).

F. Smyth is also with Pilot Photonics, Invent Centre, Dublin City University, Glasnevin, Dublin 9, Ireland.

A. D. Ellis is with Aston Institute of Photonic Technologies, Aston University, Aston Triangle, Birmingham B4 7ET, UK. optical carrier to optimize the sensitivity at the receiver.

Figure 1 shows three different possibilities to generate SCM/SSB signals with partial carrier suppression (CS). If a standard Mach Zehnder modulator (MZM) is used for the electro-optic conversion, an optical double side band signal is obtained, requiring an optical filter to suppress the undesired sideband and produce SSB. The most linear performance of this system is achieved by biasing the device at quadrature. As the optical modulation index (OMI) must be small to minimize nonlinear distortion (NLD), the carrier to signal power ratio (CPSR) is high and significant energy is wasted in the optical carrier. The power efficiency of the transmission and the sensitivity in the receiver can be improved by applying CS. A direct method to achieve CS is biasing the device at a point different to quadrature (Fig. 1(a)) although NLD will increase presenting a trade-off with the sensitivity of the link [8]. A conventional approach to generate SCM/SSB without optical filters employs a dual drive MZM (DDMZM) whose RF ports are driven by signals with a relative phase shift of 90 degrees [9]. In this case the device can only be biased at quadrature so that the optical carrier cannot be suppressed in the modulator and a costly CS block must be included [4] to increase efficiency (Fig. 1(b)). For this technique, different studies have presented comprehensive mathematical analyses of the NLD [10] and its tradeoff with CS and the sensitivity of the link [11]. An optical IQ modulator (OIQM) is an interesting device because it can generate SSB and CS without requiring additional components. In [12] it was shown that this modulator allows a wide range of CSPR values to be achieved by adjusting the bias levels (Fig 1(c)). However, no detailed investigation of NLD was carried out. As NLD increases while CSPR is reduced, a deeper analysis is required.

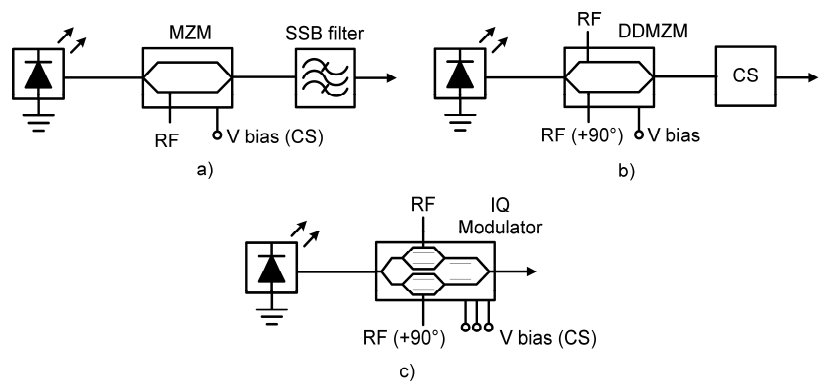

Fig. 1. Three alternatives to generate SCM/SSB/CS.

OIQMs and SCM are technologies that can be combined to achieve specific system requirements such as colorless SSB transmitters, received signals free of dispersive fading [7], 


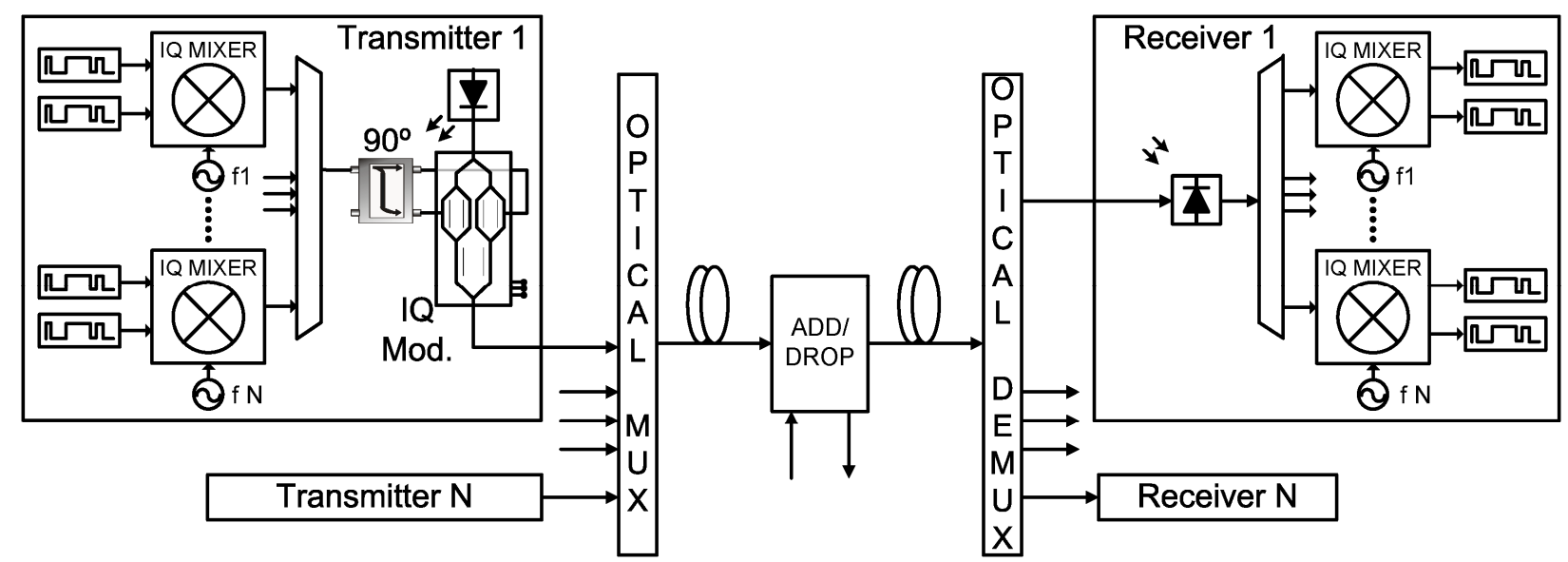

Fig. 2. WDM SCM/SSB system consisting of an optical IQ modulator and $N$ QPSK electrical subchannels per optical wavelength.

and, finally, partial optical carrier suppression. This paper studies analytically and experimentally the NLD and the CSPR that are generated simultaneously by an OIQM as a function of the bias point. The analysis presented in [12] is extended to obtain accurate CSPR and NLD for any number of RF subchannels. An optimum bias point is identified, optimizing the trade-off between NLD and CSPR in the presence of optical noise, where overall system performance is maximized. The analysis and results presented can be used to achieve efficient transmission in any multicarrier SCM link, analog or digital, without the need for a CS block. Experimental results were obtained in a $13.5 \mathrm{Gbit} / \mathrm{s}$ SCM/SSB system designed with subcarriers located at multiples of the data rate so that the distortion due to in-band intermodulation products falls in the center of other subchannels. Measurements show CSPR and NLD, their impact on the performance of channels and real gains in sensitivity. The experimental results show good agreement with theory.

\section{NONLINEAR DISTORTION AND CARRIER TO SIGNAL POWER RATIO IN OPTICAL IQ MODULATORS}

A generic SCM/SSB/WDM scheme based on OIQMs is shown in Fig. 2. In every optical wavelength, $N$ QPSK electrical subchannels are multiplexed and transmitted.

In this section, the main frequency components at the output of the OIQM will be derived. CSPR and NLD will be generalized for any frequency plan. The analytical study is based on continuous waves instead of real data as this approach has been widely used and its validity proved [13], producing good agreement with experimental data using independently modulated subcarriers.

\section{A. Introduction}

Initially, some basic concepts that will be employed in section II are reviewed. Firstly, the bias point of a MZM is determined by the total relative phase shift that is applied to the optical wavelength in the modulator arms before recombination. With a push-pull configuration a total relative phase shift equal to $2 \phi$ is obtained by producing opposite delays $(\phi$ and $-\phi)$ in each arm. Thus the bias point is defined as the absolute value of the phase shift $\phi$ introduced in each arm by the bias voltage $V_{B}$ :

$$
\phi=\frac{\pi \cdot V_{B}}{2 V_{\pi}}
$$

where $V_{\pi}$ is the half-wave voltage of the MZM. When $\phi=0$ the MZM is biased at peak, when $\phi=\pi / 4$ the device is biased at quadrature and finally when $\phi=\pi / 2$ the modulator is biased at null. The amplitude of the signal at the RF port of the MZM determines the optical modulation index (OMI). The root mean square (rms) OMI of a modulating signal composed of one subcarrier of amplitude $V_{A C}$ is given by [10]:

$$
m=\frac{\pi \cdot V_{A C}}{2 V_{\pi}} .
$$

Secondly, NLD can be divided into two groups: harmonic distortion (HD) and intermodulation distortion (IMD). The most significant are second and third order ones: $\mathrm{HD}_{2}, \mathrm{HD}_{3}$, $\mathrm{IMD}_{2}, \mathrm{IMD}_{3}$ (mixing of two frequencies) and $\mathrm{IMD}_{3 \mathrm{~B}}$ (mixing of three frequencies or triple-beat). These terms show the power of an individual distortion normalized to the power of a desired tone. Every subchannel is interfered by a different number of individual intermodulation products of each kind, $N_{C S O}$ for $\mathrm{IMD}_{2}$ and $N_{C T B}$ for $\mathrm{IMD}_{3 \mathrm{~B}}$, being its associated overall distortion referred as composite second order (CSO) and composite triple beat (CTB) respectively. CSO and CTB are the most limiting distortions in direct detected (DD) systems $[10,14]$. In general $N_{C S O}$ is higher for the lowest frequency channels and $N_{\text {СТВ }}$ is higher for the channels in the middle of the frequency plan [14].

Finally, a reference frequency plan is defined. The general theoretical results will be particularized for this case so that theory and experiments can be linked. This plan consists of five electrical subcarriers at the second, third, fourth, fifth and sixth harmonic of a fundamental frequency $(2.7 \mathrm{GHz}$ in the case of the experimental results). The first subcarrier is the most affected by $\operatorname{CSO}\left(N_{C S O}=3, N_{C T B}=2\right)$ while for a five subcarrier system the fourth (middle) subcarrier is only affected by CTB $\left(N_{C S O}=0, N_{C T B}=4\right)$. 


\section{B. Theoretical model}

As shown in Fig. 1(b) an OIQM consists of three MZMs: two parallel sub-MZMs are modulated by electrical data and the third one establishes a phase shift between the optical outputs of the two parallel sub-MZMs before recombination. Provided that the electrical inputs present a relative phase shift of 90 degrees, SSB is achieved by biasing the third MZM to produce an optical relative phase shift of 90 degrees, and the two parallel sub-MZMs at the same bias point $\phi$. These are operating conditions that are employed in the following analysis.

The study of the CSPR requires the derivation of the mathematical expression of the electrical field $E$ at the output of the OIQM. An analysis based on phasors and Bessel expansions can be used to obtain all the frequency components of the electric field $E$, for any number of RF subcarriers $N$. The following results were obtained with the phases configured to produce optical lower side band and with equal values of OMI per subcarrier $m$. The frequency of the optical carrier is denoted as $\omega_{c}$. The frequencies and phases of the $N$ subcarriers are written as $\Omega_{1}, \Omega_{2}, \ldots, \Omega_{N}$ and $\theta_{1}, \theta_{2, \ldots, \theta_{N}}$ respectively. A single expression can be used to calculate the amplitude of any fundamental tone, harmonic or intermodulation product $E_{k 1, k 2 \ldots k N}$ whose frequency is $\left(\omega_{c}+k_{1} \Omega_{1}+k_{2} \Omega_{2}+\ldots .+k_{N} \Omega_{N}\right)$, where $k_{1}, k_{2}, \ldots, k_{N}$ are arbitrary integer numbers reflecting the nature of the signal in question. This contribution is given by:

$$
\begin{gathered}
E_{k_{1}, k_{2}, \ldots, k_{n}}=E_{i} \\
\cdot\left(\prod_{i=1}^{N} J_{k_{i}}(m)\right) \cdot \cos \left(\frac{\pi}{4}\left(1+\sum_{i=1}^{N} K_{i}\right)\right) \cdot \cos \left(\phi+\frac{\pi}{2}\left(\sum_{i=1}^{N} K_{i}\right)\right) \\
\cdot \prod_{i=1}^{N} e^{j\left(k_{i}\left(\theta_{i}-\frac{\pi}{4}\right)\right)}
\end{gathered}
$$

where $J_{n}(m)$ stands for the $n$th order Bessel function of the first kind and $E_{i}$ is the amplitude of the optical carrier at the input of the OIQM. To calculate the total contribution at a desired frequency it is necessary to add all the components that fall on it. For the reference frequency plan studied here, the contribution of the first fundamental tone at $\left(\omega_{c}-\Omega_{l}\right)$ would be $E_{-1,0,0,0,0}$. While the contribution produced by $\left(\omega_{c}+\Omega_{3}-\Omega_{5}\right)$, which would fall on $\left(\omega_{c}-\Omega_{1}\right)$, would be given by $E_{0,0,1,0,-1}$. Proceeding in that way, the individual nonlinear distortions can be calculated and are shown in Table I.

For a direct detection scheme envisaged here it is necessary to obtain the distortion in the detected photocurrent $I$, which is proportional to the square of the envelope of the electrical field and is given by:

$$
I=\frac{E_{i}^{2}}{8}\left(1+\frac{1}{2}(\cos (2 m \cdot s(t)+2 \phi)+\cos (2 m \cdot \hat{s}(t)+2 \phi))\right)
$$

where $s(t)$ is equal to the sum of all the tones (normalized with amplitude 1) that are applied to one RF port and $\hat{s}(t)$ is its Hilbert-transformed pair $(s(t)$ shifted 90 degrees) that is applied to the other RF port. Again, Bessel expansions can be used to obtain all the frequency components and deduce the nonlinear distortions. They are shown in Table II. Due to the nonlinearity of the photodiode, $\mathrm{HD}_{2}$ disappears while certain $\mathrm{IMD}_{3 \mathrm{~B}}$ appears balancing all the combinations of subcarriers that give rise to triple beat.

TABLE I

NORMALIZED NLD POWER OF THE FIELD AT THE MODULATOR OUTPUT ( $\Omega_{I}, \Omega_{J}$ AND $\Omega_{K}$ ARE THREE ARBITRARY SUB CARRIER FREQUENCIES)

\begin{tabular}{c|c|c}
\hline \hline $\begin{array}{c}\text { Decond harmonic } \\
\left(\mathrm{HD}_{2}\right)\end{array}$ & $\omega_{c} \pm 2 \Omega_{i}$ & {$\left[\frac{J_{2}(m)}{\sqrt{2} \cdot J_{1}(m)} \cot \phi\right]^{2}$} \\
\hline $\begin{array}{c}\text { Third harmonic } \\
\left(\mathrm{HD}_{3}\right)\end{array}$ & $\omega_{c}+3 \Omega_{i}$ & {$\left[\frac{J_{3}(m)}{J_{1}(m)}\right]^{2}$} \\
\cline { 2 - 3 } & $\omega_{c}-3 \Omega_{i}$ & 0 \\
\hline $\begin{array}{c}2^{\text {nd }} \text { order IM } \\
\left(\mathrm{IMD}_{2}\right)\end{array}$ & $\omega_{c} \pm \Omega_{i} \pm \Omega_{j}$ & {$\left[\frac{J_{1}(m)}{\sqrt{2} \cdot J_{0}(m)} \cot \phi\right]^{2}$} \\
\hline \multirow{2}{*}{$\begin{array}{c}\text { rd } \text { order IM } \\
\left(\mathrm{IMD}_{3}\right)\end{array}$} & $\omega_{c}+\Omega_{i} \pm 2 \Omega_{j}$ & 0 \\
\cline { 2 - 3 } & $\omega_{c}-\Omega_{i} \pm 2 \Omega_{j}$ & {$\left[\frac{J_{1}(m)}{J_{0}(m)}\right]^{4}$} \\
\hline \multirow{2}{*}{$\begin{array}{c}\text { Triple beat } \\
\left(\mathrm{IMD}_{3 \mathrm{~B}}\right)\end{array}$} & $\omega_{c}+\Omega_{i}+\Omega_{j}+\Omega_{k}$ & 0 \\
\cline { 2 - 3 } & $\omega_{c}+\Omega_{i}-\Omega_{j}-\Omega_{k}$ & \\
\hline
\end{tabular}

Formulas valid for $\mathrm{m} \ll<1$

\begin{tabular}{|c|c|c|}
\hline Distortion type & Distortion frequency & Formula \\
\hline $\begin{array}{l}\text { Second harmonic } \\
\left(\mathrm{HD}_{2}\right)\end{array}$ & $2 \Omega_{i}$ & 0 \\
\hline $\begin{array}{l}\text { Third harmonic } \\
\qquad\left(\mathrm{HD}_{3}\right)\end{array}$ & $3 \Omega_{i}$ & {$\left[\frac{\left.J_{3}(2 m)\right)}{J_{1}(2 m)}\right]^{2}$} \\
\hline \multirow[t]{2}{*}{$\begin{array}{l}2^{\text {nd }} \text { order IM } \\
\qquad\left(\mathrm{IMD}_{2}\right)\end{array}$} & $+\Omega_{i}-\Omega_{j}$ & {$\left[\sqrt{2} \frac{J_{1}(2 m)}{J_{0}(2 m)} \cot 2 \phi\right]^{2}$} \\
\hline & $+\Omega_{i}+\Omega_{j}$ & 0 \\
\hline $\begin{array}{l}3^{\text {rd }} \text { order IM } \\
\left(\mathrm{IMD}_{3}\right)\end{array}$ & $\Omega_{i} \pm 2 \Omega_{j}$ & {$\left[\frac{J_{2}(2 m)}{J_{0}(2 m)}\right]^{2}$} \\
\hline $\begin{array}{l}\text { Triple beat } \\
\left(\mathrm{IMD}_{3 \mathrm{~B}}\right)\end{array}$ & $\Omega_{i} \pm \Omega_{j} \pm \Omega_{k}$ & {$\left[\frac{J_{1}(2 m)}{J_{0}(2 m)}\right]^{4}$} \\
\hline
\end{tabular}

TABLE II

NORMALIZED NLD POWER FOLLOWING PHOTO-DETECTION

( $\Omega_{I}, \Omega_{J}$ AND $\Omega_{K}$ ARE THREE ARBITRARY SUB CARRIER FREQUENCIES)

Formulas valid for $\mathrm{m} \ll<1$

\section{CSPR as a function of the bias point}

In any direct detected SCM system, the CSPR will depend on the number of electrical subchannels $N$ and the OMI. From (3), CSPR can be calculated including all the significant contributions from the fundamental tones and distortions. The accurate CSPR was obtained for the reference frequency plan and is shown in Fig. 3 for two different values of $m$. A simplified model, neglecting NLD, can be used to obtain an approximate value considering only fundamental tones:

$$
C S P R \approx \frac{E_{C}^{2}}{N E_{T}^{2}}=\frac{1}{2 N}\left[\frac{J_{0}(m)}{J_{1}(m)} \cot (\phi)\right]^{2}
$$


where $E_{C}$ is the electric field of the optical carrier and $E_{T}$ is the electric field of any fundamental tone. This approximation has been employed previously [12] but its accuracy was not analyzed. Table I shows that in the electric field $E$ the main contributions of NLD come from $\mathrm{HD}_{2}$ and $\mathrm{IMD}_{2}$, being maximum at peak bias point and canceling while approaching the null. For that reason the approximated CSPR, which is also shown in Fig. 3, diverges clearly around peak. However, for the bias points of interest in DD systems, between quadrature and $n u l l$, the approximation is valid, especially for low values of $m$ (error less than $0.3 \mathrm{~dB}$ from quadrature in this example).

The curves in Fig. 3 also show the CSPR that is obtained with the most significant terms of $I$ obtained from (4). Apart from the area around the null point, CSPR can be approximated by measuring the detected photocurrent and adding $3 \mathrm{~dB}$. This technique will be used to measure CSPR in the experimental section as direct measurement is not possible.

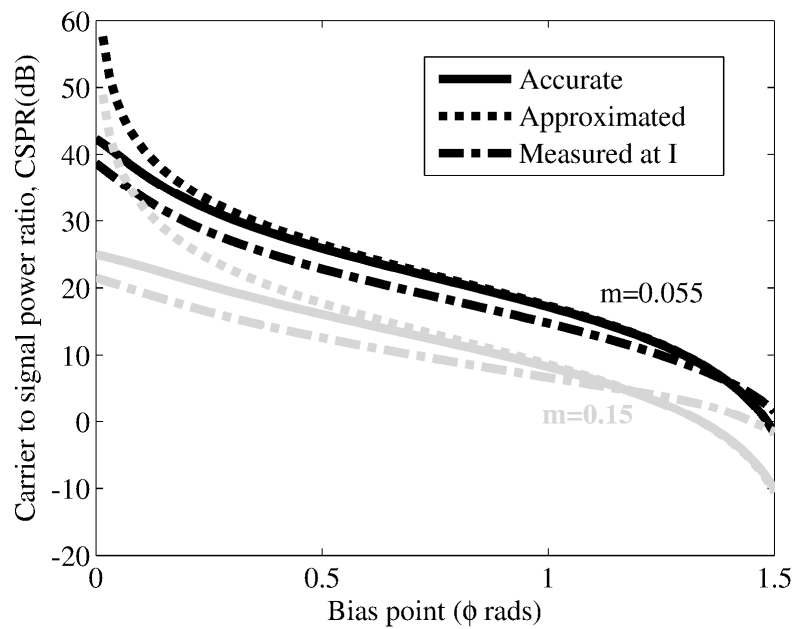

Fig. 3. Accurate and approximated CSPR for an SCM/SSB system composed of an optical IQ modulator and five subcarriers for two different values of subchannel OMI (0.055 and 0.15).

It can be concluded that, as expected, CSPR decreases when the bias point moves towards null. Different bias points produce different partial carrier suppression while the power of the transmitted tones remains the same. Thus decreasing the CSPR, and neglecting NLD, the subchannels could be received with the same quality with a lower received average optical power, thereby improving sensitivity. However, as it is explained in the next section, some subchannels can be severely impaired due to NLD when the bias point changes.

\section{NLD as a function of the bias point}

From Table II it can be confirmed that the most limiting NLD in the detected photocurrent $I$ are second order intermodulation and triple beat (CSO and CTB when all the intermodulation products are considered). Fig. 4 shows both distortions for two values of OMI. Whereas $\mathrm{IMD}_{3 \mathrm{~B}}$ depends only on $m, \mathrm{IMD}_{2}$ varies with the bias point cancelling at quadrature and worsening sharply if the bias point changes. In both cases higher values of $m$ give rise to higher distortions.

The previous results have illustrated the combined behavior of CSPR and NLD as a function of the bias point. Both terms present a relationship that can be manifested in two different ways depending on the nature of the IMD. All the cases can be divided into two groups: systems where the desired tones are only interfered by CTB and systems where at least one of the subchannels is interfered by CSO. The first case happens when the frequency plan ensures that $\mathrm{CSO}$ is not interfering $\left(N_{C S O}=0\right.$ for all the electrical subcarriers), thus NLD is composed only of CTB and is constant irrespectively of the bias point. This is achieved when there is a guard band between the optical carrier and the first subcarrier equal to at least half of the total spectrum as in DD/OFDM [15] or some CATV schemes [16]. This particular case is simpler to analyse and for a given OMI the optimum bias point would be obtained when CSPR is equal to one, as it has been experimentally shown [17]. Strong clipping appears in the signal but it does not have a detrimental effect because its associated distortion translates into CSO and falls in the part of the spectrum where no desired subcarriers are present. A deeper analysis, like the one presented in the next section would be required to find the optimum OMI. The second case happens when there are low frequency subcarriers as in broadband SCM [4] or in the reference example. In this case there is a trade-off between CSPR and NLD: biasing closer to null improves CSPR but at the same time CSO increases sharply. A mathematical analysis of the system is required to obtain the sensitivity at the receiver as a function of the bias point so that the optimum bias can be found.

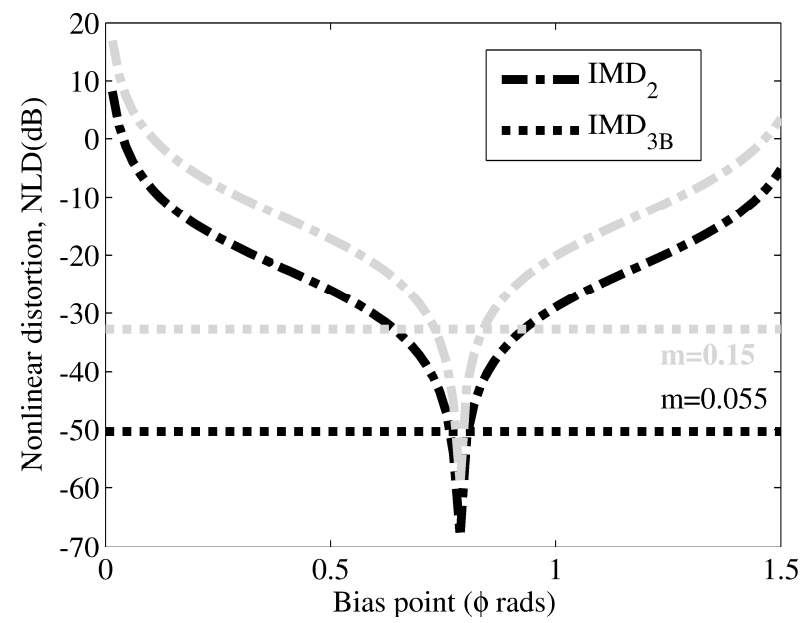

Fig. 4. Individual NLD ( $\mathrm{IMD}_{2}$ and $\left.\mathrm{IMD}_{3 \mathrm{~B}}\right)$ at the detected photocurrent of an SCM/SSB system using an optical IQ modulator for two values of OMI.

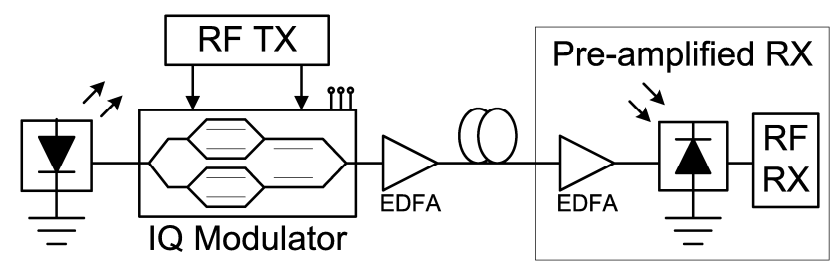

Fig.5. SCM/SSB scheme with a pre-amplified optical receiver.

\section{E. Optimum bias point}

The study was based on an SCM link composed of $N$ 
subcarriers and with a pre-amplified receiver as shown in Fig. 5. This receiver consists of an erbium doped fiber amplifier (EDFA), a photo-detector and an RF demodulator.

The average optical power $P_{I N}$ at the input of the receiver required for a given quality factor $Q_{F}$ is mathematically derived in the Appendix A:

$$
P_{I N}=\frac{4 Q_{F}^{2} F h v B_{e}}{I_{\phi}^{2}\left(1-Q_{F}^{2}\left(N_{C S O} I M D_{2}+N_{C T B} I M D_{3 B}\right)\right)} .
$$

where $F$ is the noise figure of the EDFA, $h$ is Planck's constant, $v$ is the optical frequency and $B_{e}$ is the electrical bandwidth of the baseband channel at the receiver. $I_{\phi}$ represents the dependency of the amplitude of the subcarrier on the bias point:

$$
I_{\phi}=\frac{\sqrt{2} J_{0}^{N-1}(2 m) J_{1}(2 m) \sin (2 \phi)}{1+J_{0}^{N}(2 m) \cos (2 \phi)} .
$$

As the only sources of noise and distortion considered are ASE and NLD, $P_{I N}$ in eq. (6) represents the minimum sensitivity that is theoretically achievable in a QPSK SCM/SSB link implemented with an OIQM and a preamplified receiver.

The gain in sensitivity that can be achieved by varying the bias point depends on NLD. This distortion is different for every subchannel because it is determined by different values of $N_{C S O}$ and $N_{\text {CTB }}$. Therefore, depending on the nature of the link, the optimum bias point is given by either the average bit error rate (BER) or the BER of the worst case channel. The second condition is the strictest and is used in this analysis.

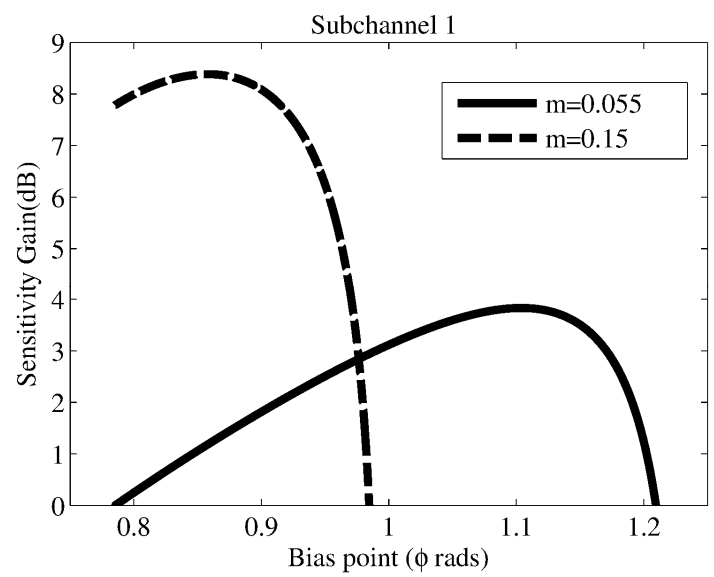

Fig. 6. Gains in sensitivity for $Q_{F}=6$ with respect to quadrature and $m=0.055$ for a subchannel distorted by NLD $\left(\mathrm{N}_{C S O}=3\right.$ and $\left.N_{C T B}=2\right)$ in a five subcarrier QPSK SCM/SSB link consisting on an optical IQ modulator and a preamplified receiver $\left(\mathrm{F}=5 \mathrm{~dB}, v=193.4 \mathrm{THz}, B_{e}=2.7 \mathrm{GHz}\right)$

These results will be particularized for the reference design considering only subcarrier 1 , as it is the most affected by CSO $\left(N_{C S O}=3\right.$ and $\left.N_{C T B}=2\right)$, and subcarrier 4 , as it is the most interfered of the channels affected only by CTB $\left(N_{C S O}=0\right.$ and $\left.N_{C T B}=4\right)$. Considering quadrature and a low value of $m$ as the initial point, Fig. 6 shows the gains in sensitivity (for $Q_{F}=6$,
$\mathrm{BER}=10^{-9}$ ) that can be achieved changing the settings. Moving the bias point towards null, the sensitivity improves notably until CSO is comparable to the optical noise floor. For a value of $m=0.055$, there is an optimum bias point ( $\phi=1.1 \mathrm{rad})$, where a sensitivity gain of almost $4 \mathrm{~dB}$ with respect to quadrature is achieved. This gain is very sensitive to changes in $\phi$ (it is reduced to $3 \mathrm{~dB}$ at $\phi=1.175)$ and $m(3.5 \mathrm{~dB}$ with $m=0.06)$. From that point sensitivity improves while $m$ increases and the bias point moves towards quadrature. This effect occurs because CSPR improves with a higher OMI and CSO tends to cancel while close to quadrature. A gain in sensitivity of $8 \mathrm{~dB}$ is observed for $m=0.15$ with respect to $m=0.055$ at quadrature. However, while $m$ increases, the impact of CTB on subchannel 4 also increases, as shown in Fig. 7. For that channel, as CTB is constant regardless of the bias point, a gain in sensitivity is always achieved when moving the bias from quadrature towards null. For $m=0.055$, the gain in sensitivity at the optimum bias point of subchannel $1, \phi=1.1 \mathrm{rad}$, is $6 \mathrm{~dB}$ with respect to quadrature. When $m$ increases, CSPR reduces and $\mathrm{CTB}$ increases, resulting in better sensitivity until the level of CTB is comparable with the optical noise floor, as it happens at $m=0.2$. From that value, further increments of $m$ result in a loss in sensitivity.

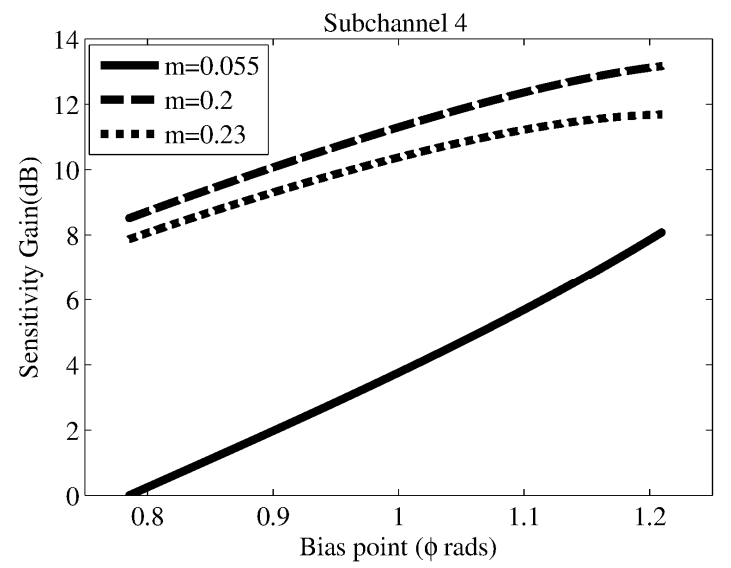

Fig. 7. Gains in sensitivity for $Q_{F}=6$ with respect to quadrature and $m=0.055$ for a subchannel distorted by $\mathrm{NLD}\left(\mathrm{N}_{C S O}=0\right.$ and $\left.N_{C T B}=4\right)$ in a five subcarrier QPSK SCM/SSB link consisting on an optical IQ modulator and a preamplified receiver $\left(\mathrm{F}=5 \mathrm{~dB}, v=193.4 \mathrm{THz}, B_{e}=2.7 \mathrm{GHz}\right)$.

The previous analysis can be applied to achieve optimum sensitivity in any SCM link using an OIQM. The influence of the bias point can be summarized with two effects. Firstly, for a given OMI, it is possible to find a bias point different to quadrature and closer to null where overall sensitivity improves. Secondly, as the OMI increases, this optimum bias point will be closer to quadrature. In the following section a real scheme is used to validate the predicted gains in sensitivity and to show the described trends. The overall link performance is determined by the combined behavior of, among others, the OMI, the bias point, and the performance of the optical and RF components in the bands of use. For different system design level objectives, different individual parameters may be required. On many occasions OMI is maintained small to guarantee low intermodulation distortion 
but this behavior can cause loss in sensitivity. This effect can be overcome with an increase in the OMI of the signal, but that requires a higher power consumption of the RF components. As power consumption is usually a major issue, a lower value of OMI can be used accompanied with a bias closer to null to achieve equivalent sensitivity

\section{EXPERIMENTAL RESULTS}

The experimental setup was a five carrier SCM/QPSK system with the electrical transmitter and receiver equivalent to that shown in Fig. 2. The IQ mixers were off-the-shelf monolithic microwave integrated circuits. SCM/QPSK has been previously reported [18] but employing two regular mixers and microwave circuitry instead of the integrated IQ mixers used in this work. The baud rate per subcarrier was $1.35 \mathrm{Gbaud}$, making a total data rate of $13.5 \mathrm{Gbit} / \mathrm{s}$ for every optical channel. An FPGA was used to generate patterns of $2^{15}$ bits. The data streams were low pass filtered to reduce the spectrum of the signal to the first lobe of a sinc function. Imitating the reference design that was studied in section II, the employed RF local oscillators (LO) were even harmonics of the data rate (from 5.4 to $16.2 \mathrm{GHz}$ ), obtained using an electrical comb and appropriate demultiplexing filters. Thus no guard band was introduced between subchannels and the total energy of any intermodulation product distorted only one subchannel. No digital signal processing (DSP) algorithms were employed to compensate the impairments of the analogue components. The electrical signal was applied to a thin film polymer OIQM [19] with a bandwidth of $20 \mathrm{GHz}$ and $V \pi$ of 2.5 Volts.

The setup was completed in two different configurations depending on the parameter to be measured. Both configurations will be described in the next sections. To make the experimental results comparable with theory $m$ has to be redefined for the case of real data. When a modulated electrical subcarrier presents an rms voltage of $V_{R M S}$ the experimental rms OMI is:

$$
m=\frac{\pi \cdot V_{R M S}}{2 V_{\pi}}
$$

It should be noted that the peak to average power ratio (PAPR) of the final signal will depend on the OMI, number of subcarriers and the particular relative phases of the subcarriers when they combine. However, theoretically, system performance will be determined by the rms power or the rms OMI of the final signal, $m \sqrt{ } N$, regardless of the individual phases and the PAPR that arises. Obviously it has to be ensured that the rest of the components in the system can handle that PAPR value without including additional nonlinearities.

\section{A. Bias points}

The system characterization was carried out by biasing the two parallel sub-MZMs of the OIQM to get equal intensity from them, and then varying these bias levels between peak and null. Sixteen levels of intensity were selected. For each bias setting of these sub-MZMs, the bias point of the third MZM (that combines the optical outputs of the parallel ones) was adjusted to achieve the best suppressed side band rejection (SSR). In Fig. 8 the optical spectrum of one of these realizations is shown, where an SSR of $20 \mathrm{~dB}$ can be observed.

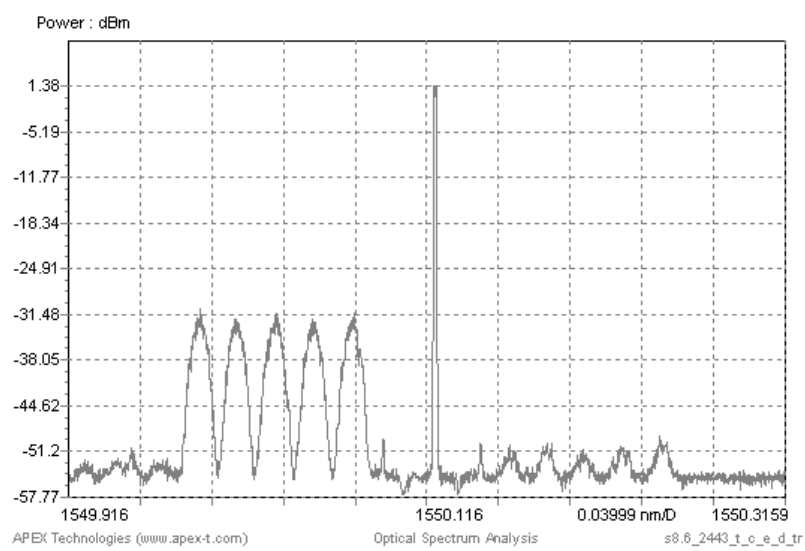

Fig. 8. Optical spectrum obtained with $\mathrm{m}=0.055$ at a one of the sixteen bias points analyzed. OLSB with an SSR of $20 \mathrm{~dB}$ is achieved. CSPR $=15.7 \mathrm{~dB}$.

\section{B. Agreement with the model: measured CSPR and IMD.}

The theoretical analysis concluded that CSPR and $\mathrm{IMD}_{2}$ are the two key parameters to determine the optimum bias point in an SCM link implemented with an OIQM. These parameters were measured experimentally to verify the agreement with the model obtained with the employed OIQM. For this particular device the bias is established with constant current instead of voltage, thus the measurements will be presented as a function of bias current. The tests were performed with the configuration shown in Fig. 9. At the output of the OIQM a variable optical attenuator (VOA) was included prior to an EDFA. The gain of the EDFA was maintained constant and the VOA attenuation was modified to ensure the same optical power at the input of the EDFA for all the bias points that were analyzed. With this method the measurements at different bias points were taken under the same conditions.

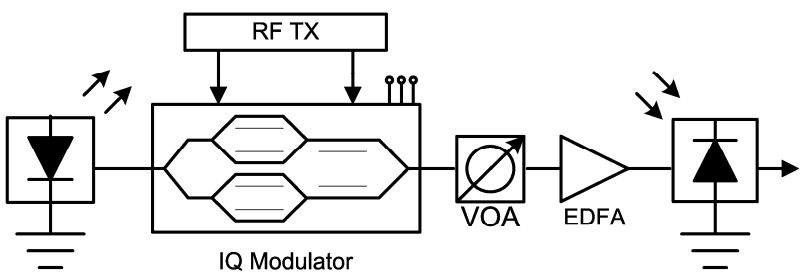

Fig. 9. Setup employed in the characterization of the optical modulator.

To investigate $\mathrm{IMD}_{2}$, a discrete photo-detector with a bandwidth of $20 \mathrm{GHz}$ was employed. Its output was connected to a spectrum analyzer. In the transmitter only the first (5.4 $\mathrm{GHz})$ and the fifth $(16.2 \mathrm{GHz})$ electrical subcarriers were activated such that the second order intermodulation product for this frequency plan falls at $10.8 \mathrm{GHz}$. Fig. 10 illustrates the measured and theoretical $\mathrm{IMD}_{2}$ that were obtained for a modulation index $m=0.15$ for the different bias points. A trace 
from the spectrum analyzer after the photo receiver is also shown. A good agreement between theory and measurements is observed. The discrepancy with the peak of the theoretical value is due to the fact that the minimum distortion that could be measured was limited by the noise floor. With lower values of OMI this limitation is present in more bias points and for that reason those measurements are not shown. The previous result confirms that biasing at a point different to quadrature translates into a meaningful increase of CSO. In the next section it will become clear that this impairment can be balanced with the associated improvement in CSPR that gives rise to overall sensitivity.

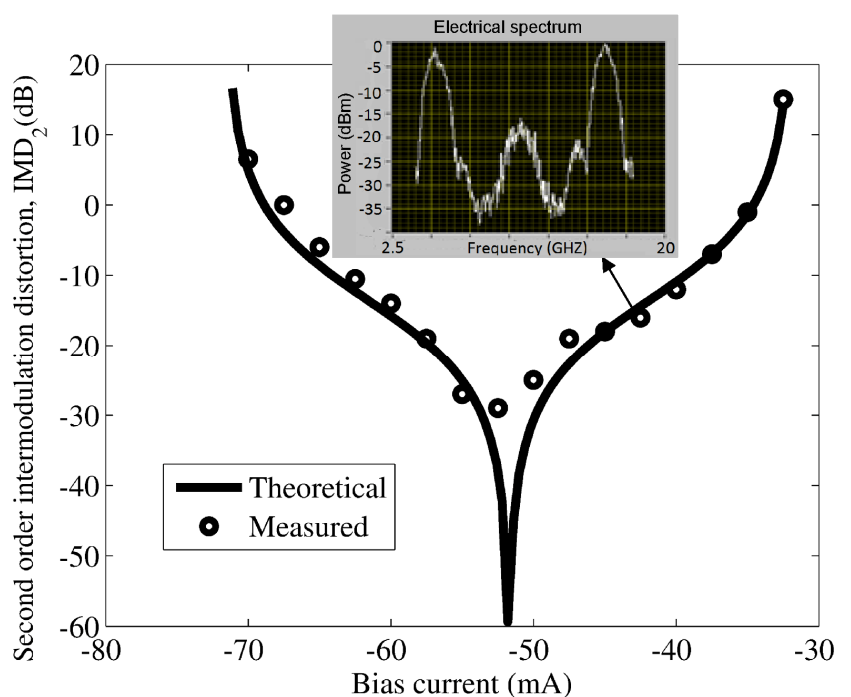

Fig.10. Second order intermodulation distortion. Measurements performed activating only the first and the fifth carrier and measuring the distortion at the third one. $m=0.15$. Electrical spectrum at one of the measurements.

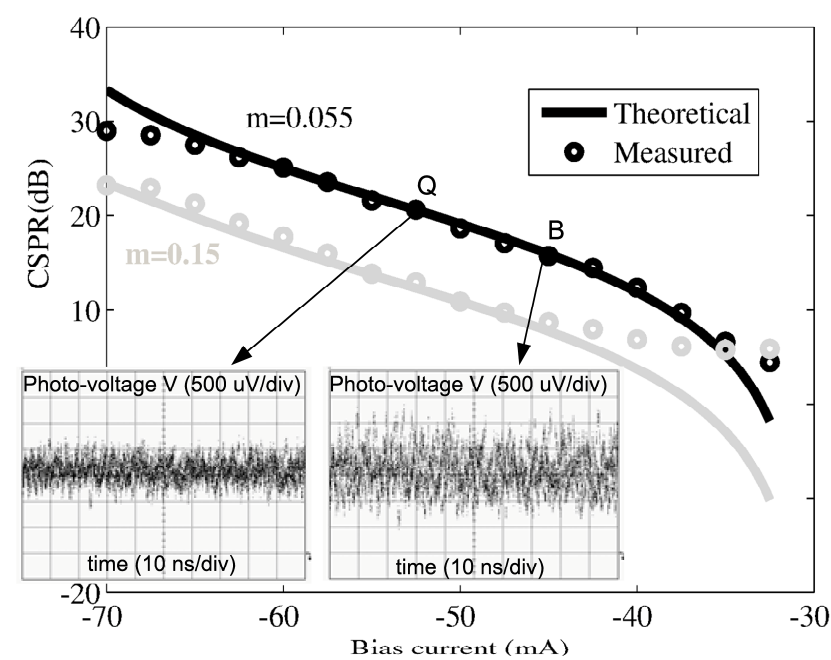

Fig. 11. CSPR: theoretical and measured. Five carriers. $m=0.055$ and 0.15 . Photocurrent at two different bias points: quadrature and point B.

For the measurement of the CSPR, the photo-detector of a digital oscilloscope was used. CSPR was obtained for the detected photocurrent as the square of the ratio between the DC component and the rms value of the AC signal. Fig. 3 showed that a good approximation of the optical CSPR could be obtained adding $3 \mathrm{~dB}$ to the value obtained with the detected photocurrent (excluding the area around null). Fig. 11 illustrates the accurate theoretical value and the measurements for $m=0.055$ and $m=0.15$. A good agreement between theory and the experiment is observed. For the case of lower OMI, the picture also shows the photocurrent at two bias points: quadrature ( $\mathrm{Q}$ in the figure), where $\mathrm{IMD}_{2}$ presented its lowest value, and point $\mathrm{B}$, the closest to the optimum that was identified in the theoretical section for $m=0.055$. The improvement in CSPR at point $\mathrm{B}$ is $5 \mathrm{~dB}$ relative to $\mathrm{Q}$, and the optical peak to peak modulation depth approaches unity (roughly from $2 / 4$ to $4 / 5$ ), making this point more suitable for an efficient transmission.

\section{Effects of CSPR and NLD on channel performance.}

This section shows how the CSPR and NLD induced by the OIQM influences the performance of electrical channels in the communications system. The results are taken with a setup similar to that shown in Fig. 5, simulating the losses in the fiber with a VOA so that dispersion effects are removed. The gain of the EDFA in the transmitter is fixed to obtain $10 \mathrm{dBm}$ at the output. The gain of the EDFA at the receiver is regulated to guarantee the sensitivity in the baseband electrical receivers irrespective of the incoming optical power. The measurements were focused on two carriers, subchannel 1 to observe the effects of CSO and subchannel 4 to analyse the effects of CTB. The modulation index was $m=0.055$ and two bias points were analyzed: $Q$ and $B$. BER versus received optical power were obtained in two different situations: activating only one electrical carrier so that no IMD was produced, and with all the carriers on and NLD present in the system.

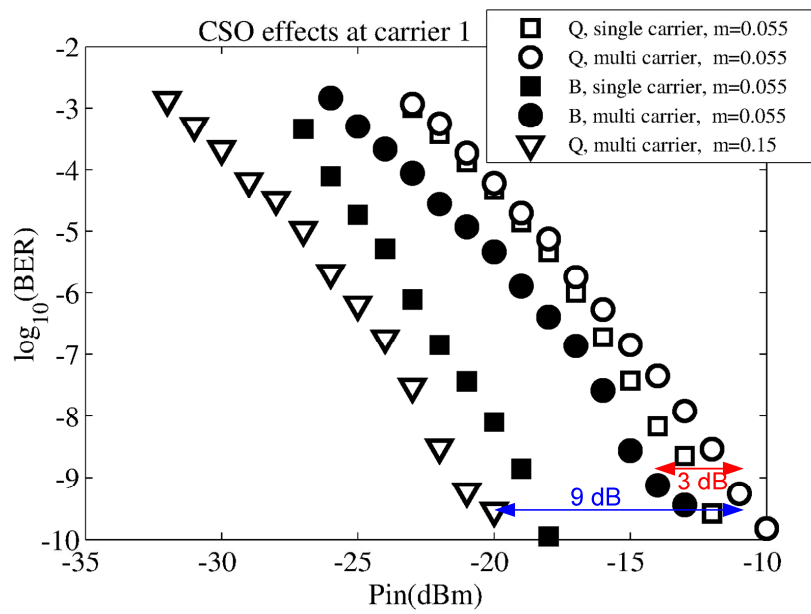

Fig. 12. BER vs. Optical input power at the receiver for the first electrical carrier. Two different bias points, $Q$ and $B$. Measurements obtained with one or five active carriers. $m=0.055$ and 0.15 .

The performance of the first subchannel can be observed in Fig. 12. As expected, the influence of the intermodulation products is more severe when biasing closer to null at point $B$. At $Q, \mathrm{CSO}$ is cancelled, so there is only a minor difference in performance when all the channels are active (less than $1 \mathrm{~dB}$ ). However, when biasing at $B$, the NLD coming from CSO causes a penalty in performance of up to $5 \mathrm{~dB}$. Despite the higher penalty due to NLD, a sensitivity gain of $3 \mathrm{~dB}$ can be observed at point $\mathrm{B}$ with respect to $\mathrm{Q}$, for a $\mathrm{BER}=10^{-9}$, in 
agreement with the theoretical prediction.

A similar analysis was done for the fourth carrier and is shown in Fig. 13. CTB is independent of the bias point, as has been illustrated in Fig. 4. Due to the low value of CTB for this OMI, the difference that can be observed between the single and multicarrier cases is small and, as expected, is similar at both bias points. The gain in sensitivity due to the better CSPR at point $\mathrm{B}$ is $6 \mathrm{~dB}$ with respect to $\mathrm{Q}$, as predicted by theory for a $\mathrm{BER}=10^{-7}$ ( similar to the prediction for a $\mathrm{BER}=10^{-9}$ ).

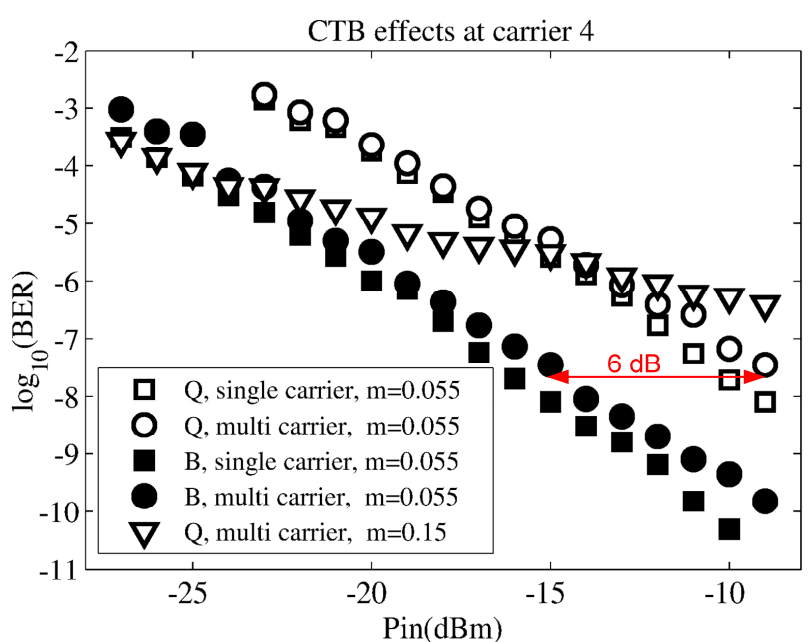

Fig. 13. BER vs. Optical input power at the receiver for the fourth electrical carrier. Two different bias points, $Q$ and $B$. Measurements obtained with one or five active carriers. $m=0.055$ and 0.15 .

\section{Effects of increased OMI.}

According to the theoretical study for OIQM-based SCM systems, the behavior of the sensitivity can be summarized with two trends. For a given OMI, there can be an optimum bias point different to quadrature, where the channel most affected by CSO determines the gain in sensitivity of the link. This effect has been verified experimentally in the previous section. The second trend shows that it is possible to improve sensitivity by increasing OMI and biasing closer to quadrature, but at some point this process will have a detrimental effect for the channels affected by CTB. This behavior is showed in this section.

The effects of CSO on subchannel 1 shown in Fig. 12 also include the performance for the case of increased OMI with $m=0.15$ biasing at quadrature. The higher value of $m$ induces a better CSPR and the bias at quadrature cancels CSO, resulting in a gain in sensitivity of $9 \mathrm{~dB}$ with respect to the lower OMI, consistent with the $8 \mathrm{~dB}$ predicted by theory. On the other hand, the behavior of channel 4 shown in Fig. 13 includes the same case of increased OMI while biasing at quadrature. It can be seen that the increment in CTB associated to the higher OMI resulted in a loss of sensitivity with respect to the case of lower OMI for all BER below $10^{-5}$. This penalty occurred with an OMI lower than that expected from the theoretical prediction $(m=0.20)$. This is due to the fact that the model included ideal electrical IQ mixers. In practice, as these components are designed for lower bandwidth signals [20], the use of higher rates translates into a significant eye closure that can appear like increased NLD.

\section{CONCLUSIONS}

SCM is a reliable multicarrier technique that is employed in multiple schemes, usually combined with SSB to obtain increased tolerance to dispersive fading. The mathematical study of SCM has been extended by analyzing the OIQM, an ideal choice in the transmitter because, unlike other alternatives, both SSB and carrier suppression can be obtained directly without external components by adjusting the bias points.

When the frequency plan ensures that the subchannels are only affected by CTB, CSPR can be modified without adding any impairment in the subchannels. When at least one of the subchannels is interfered by CSO, a trade-off between CSPR and NLD is present. The developed mathematical model can be used to determine the optimum bias point and predict the gains in sensitivity that can be achieved for every subchannel.

Experiments have been conducted with a scheme and components that ensure subcarriers are located at multiples of the data rate, which makes it ideal for an accurate study of the effects of intermodulation distortion. The theoretical predictions have been validated measuring CSPR, NLD, and their effect on the performance of channels. The sensitivity gains that can be directly achieved with OIQM prove the suitability of this device to improve the power budget of SCM/SSB links without incurring additional costs.

\section{APPENDIX A}

This appendix shows the mathematical derivation of the sensitivity required for a desired quality factor $Q_{F}$ in a SCM/SSB link composed of an optical IQ modulator, a preamplified receiver (see Fig. 5) and $N$ QPSK subcarriers.

This receiver consists of an erbium doped fiber amplifier (EDFA) whose gain is $G$, a photo-detector whose responsivity is $R$ and an RF demodulator. The average optical power at the input of the receiver can be obtained as the DC component of the photocurrent detected with a responsivity of 1 . From (4):

$$
P_{I N}=I_{D C}=\frac{1}{8}\left(1+J_{0}^{N}(2 m) \cos (2 \phi)\right)
$$

where $E_{i}$ has been normalized to 1 . The amplitude of the $k$ th electrical subcarrier at the detected photocurrent can also be obtained from (4):

$$
A_{k}=R G \frac{\sqrt{2}}{8} J_{0}^{N-1}(2 m) J_{1}(2 m) \sin (2 \phi) .
$$

To calculate the sensitivity of the link, the detected photocurrent must be rewritten making $P_{I N}$ an independent variable:

$$
I=R G P_{I N}\left(1+\sum_{k=1}^{N} I_{\phi} \cos \left(\Omega_{k} t+\theta_{k}\right)+N L D\right)
$$

where $\Omega_{k}$ and $\theta_{k}$ are the frequency and phase of the subcarrier 
and $I_{\phi}$ represents the dependency of the amplitude of the subcarrier on the bias point:

$$
I_{\phi}=\frac{\sqrt{2} J_{0}^{N-1}(2 m) J_{1}(2 m) \sin (2 \phi)}{1+J_{0}^{N}(2 m) \cos (2 \phi)} .
$$

Thus, the amplitude of the $k$ th subcarrier can be written as:

$$
I_{k}=R G P_{I N} I_{\phi}
$$

In the described link, neglecting NLD, the main source of noise will be amplified spontaneous emission (ASE) coming from the EDFA. After demodulation the contribution of this noise will be [4]:

$$
\sigma_{A S E}^{2}=2 R^{2} P_{I N} G(G-1) F h v B_{e}
$$

where $F$ is the noise figure of the EDFA, $h$ is Planck's constant, $v$ is the optical frequency and $B_{e}$ is the electrical bandwidth of the baseband channel at the receiver. Under a Gaussian approximation the time averaged second and third order intermodulation current power $[21,22]$ is added like noise as in [11]. Thus the absolute contribution of NLD is:

$$
\sigma_{N L D}^{2}=\sigma_{C S O}^{2}+\sigma_{C T B}^{2}=\left(N_{C S O} \frac{I M D_{2}}{2}+N_{C T B} \frac{I M D_{3 B}}{2}\right) I_{K}^{2} .
$$

The quality factor $Q_{F}$ after the RF demodulator for a QPSK SCM system is obtained with the following expression [4]:

$$
Q_{F}=\frac{\sqrt{2} I_{K}}{2\left(\sqrt{\sigma_{A S E}^{2}+\sigma_{N L D}^{2}}\right)} .
$$

After some manipulations and for $G>>1$, the sensitivity for a desired value of $Q_{F}$ can be deduced as:

$$
P_{I N}=\frac{4 Q_{F}^{2} F h v B_{e}}{I_{\phi}^{2}\left(1-Q_{F}^{2}\left(N_{C S O} I M D_{2}+N_{C T B} I M D_{3 B}\right)\right)} .
$$

\section{REFERENCES}

[1] P. M. Hill and R. Olshansky, "A 20-channel optical communication using subcarrier multiplexing for the transmission of digital video signals," J. Lightwave Technol., vol. 8, pp. 554-560, Apr. 1990

[2] G. H. Smith and D. Novak, "Broad-band millimeter-wave (38 GHz) fiber-wireless transmission system using electrical and optical SSB modulation to overcome dispersion effects," IEEE Photon. Technol. Lett., vol. 10, pp. 141-143, Jan. 1998.

[3] R. P. Almedia, R. S. Oliveira, N. S. Moritsuka, C. R. L. Frances, A. Teixeira, and J. C. W. A. Costa, "Digital Radio over Fibre transmission based on SCM and WDM system for C-Ran architecture" in Telecommunications Symposium (ITS), pp 1-5, Sao Paulo, 2014.

[4] R. Hui, B. Zhu, R. Huang, C. T. Allen, K. R. Demarest, and D. Richards, "Subcarrier multiplexing for high-speed optical transmission," J. Lightw. Technol., vol. 20, no. 3, pp. 417-427, Mar. 2002.

[5] B. Charbonnier, S. Menezo, P. O'Brien, A. Lebreton, J.M. Fedeli and B. Ben Bakir, "Silicon photonics for next generation FDM/FDMA PON," J. Opt. Commun. Netw., vol. 4, no. 9, pp. A29-A37, (2012).

[6] M. Salter, D. Platt, L. Petterson, L. Aspemyr, M. Bao, "Circuits and systems simulations for $100 \mathrm{~Gb} / \mathrm{s}$ optical SCM transmission", ICECS 2009, pp 960-963.

[7] J. Maeda, T. Kato and S. Ebisawa, "Effect of fiber dispersion on subcarrier QAM signal in radio-over-fiber transmission," J. Lightwave Technol., vol. 30, no. 16, pp. 2625-2632, Aug. 2012.

[8] J. Leibrich, A. Ali, and W. Rosenkranz, "OFDM transceiver design for optimizing sensitivity and long-haul performance," in Proc. IEEE LEOS Summ. Top. 2008, pp. 249-250, Paper WD3.1.

[9] G. H. Smith, D. Novak, and Z. Ahmed, "Overcoming chromatic dispersion effects in fiber-wireless systems incorporating external modulators," IEEE Trans. Microwave Technol., vol. 45, pp. 1410-1415, Aug. 1997.

[10] W. H. Chen and W. I. Way, "Multichannel single-sideband SCM/DWDM transmission systems," IEEE Journal of Lightwave Technology, vol. 22, no. 7, pp. 1679-1693, 2004.

[11] P. Laurencio, S. O. Simoes, and M. C. R. Medeiros, "Impact of the combined effect of RIN and intermodulation distortion on OSSB/SCM systems," J. Lightw. Tech., vol. 24, no. 11, pp. 4250-4262, Nov. 2006.

[12] T. Nakatogawa, M. Maeda and K. Oyamada, "Optical single sideband modulator for distribution of digital broadcasting signals on millimetrewave band based on self-heterodyne," Electron. Lett., vol. 40, no. 21, pp. 1369-1370, 14th October 2004.

[13] M.-C. Wu, P.-Y. Chiang, and W. I. Way, "On the validity of using CW tones to test the linearity of multichannel M-QAM subcarrier multiplexed lightwave systems," IEEE Photon. Technol. Lett., vol. 12, pp. 413-415, Apr. 2000.

[14] W. I. Way, Broadband Hybrid Fiber/Coax Access System Technologies. New York: Academic, 1998.

[15] B. J. C. Schmidt, A. J. Lowery, and J. Armstrong, "Experimental demonstrations of electronic dispersion compensation for long-haul transmission using direct-detection optical OFDM," J. Lightw. Technol., vol. 26, no. 1, pp. 196-203, Jan. 2008.

[16] T. Nakatogawa, M. Maeda and K. Oyamada, "A novel millimeter wave receiver using self-heterodyne detection for a digital broadcasting ROF system," presented at the Microw. Photon. '06, Grenoble, France, Oct. 2006.

[17] A. J. Lowery and J. Armstrong, "Orthogonal-frequency-division multiplexing for dispersion compensation of long-haul optical systems," Opt. Express, vol. 14, no. 6, pp. 2079-2084, Mar. 2006.

[18] J. Y. Ha, A. Wonfor, R. V. Penty, I. H.White, and P. Ghiggino, "Spectrally efficient $10 \times 1 \mathrm{~Gb} / \mathrm{s}$ QPSK multi-user optical network architecture," presented at the OFC 2007, Anaheim, CA, OWD6.

[19] G. Yu, E. Miller, J. Mallari, C. Wei, B. Chen, H. Chen, V. Shofman and R. Dinu, "Small form factor thin film polymer modulators for telecom applications," in Proc. OFC, 2012, pp. 1-3, paper OM3J.

[20] B. E. Olsson, J. Mårtensson, A. Kristiansson, and A. Alping, "RF Assisted Optical Dual-Carrier 112 Gbit/s Polarization- Multiplexed 16QAM Transmitter," Proc. OFC, San Diego, paper OMK5, 2010.

[21] R. Olshansky, "Optimal design of subcarrier multiplexed lightwave systems employing linearized external modulators," J. Lightw. Technol., vol. 10, no. 3, pp. 378-382, Mar. 1992.

[22] F. Ramos and J. Marti, "Compensation for fiber-induced composite second-order distortion in externally modulated lightwave AM-SCM systems using optical-phase conjugation," J. Lightw. Technol., vol. 16, no. 8 , pp. 1387-1392, Aug. 1998 . 